UCRL-ID- 121728

\title{
Comments on the Kinetic Dynamo
}

\author{
T. K. Fowler
}

August 24, 1995

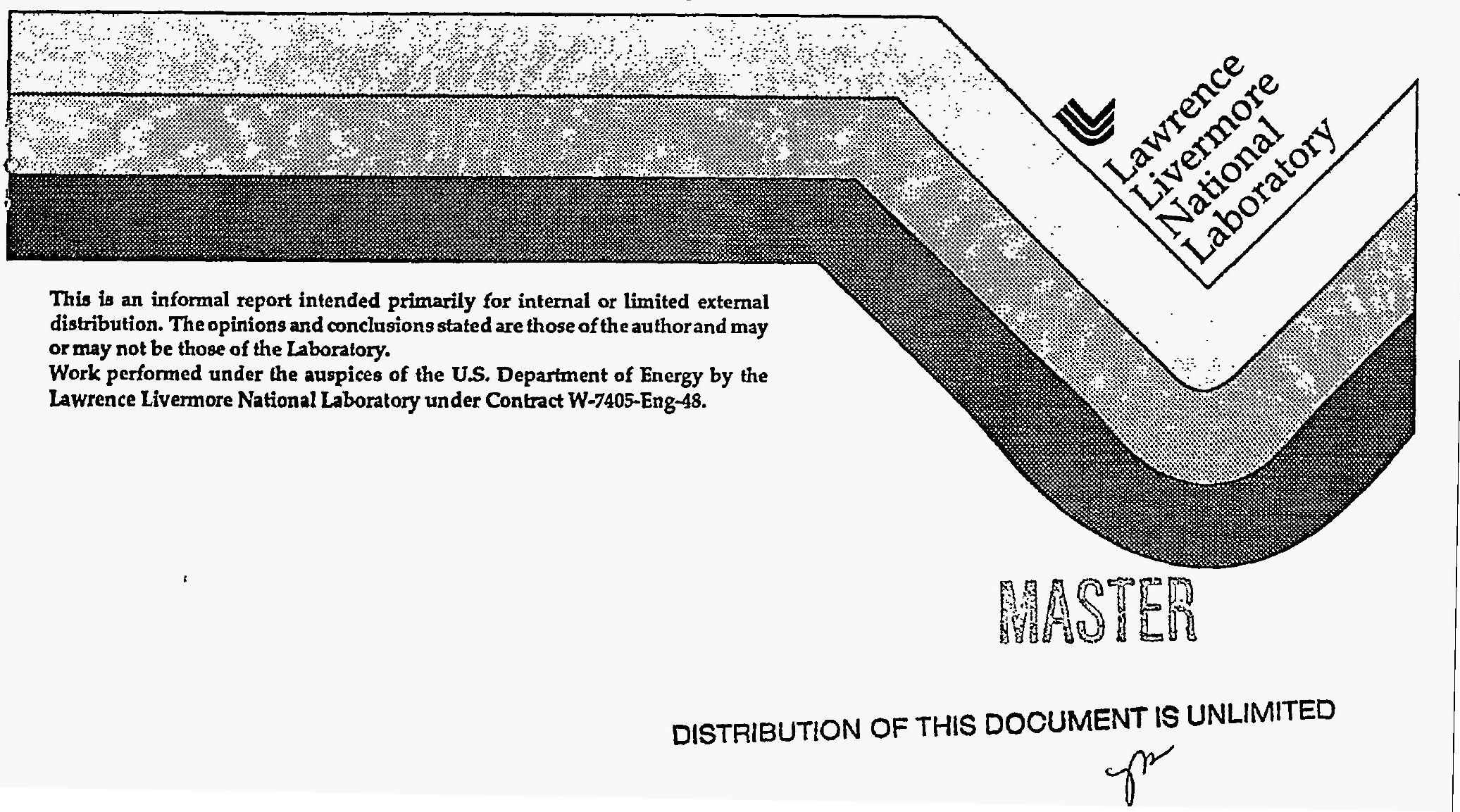




\section{DISCLAIMER}

This document was prepared as an account of work sponsored by an agency of the United States Government. Neither the United States Government nor the University of California nor any of their employees, makes any warranty, express or implied, or assumes any legal liability or responsibility for the accuracy, completeness, or usefulness of any information, apparatus, product, or process disclosed, or represents that its use would not infringe privately owned rights. Reference herein to any specific commercial product, process, or service by trade name, trademark, manufacturer, or otherwise, does not necessarily constitute or imply its endorsement, recommendation, or favoring by the United States Government or the University of California. The views and opinions of authors expressed herein do not necessarily state or reflect those of the United States Government or the University of California, and shall not be used for advertising or product endorsement purposes.

This report has been reproduced directly from the best available copy.

Available to DOE and DOE contractors from the Office of Scientific and Technical Information P.O. Box 62, Oak Ridge, TN 37831

Prices available from (615) 576-8401, FTS 626-8401

Available to the public from the National Technical Information Service

U.S. Department of Commerce 5285 Port Royal Rd. Springfield, VA 22161 


\section{DISCLAIMER}

Portions of this document may be illegible in electronic image products. Images are produced from the best available original document. 


\title{
Comments on the Kinetic Dynamo
}

\author{
T. K. Fowler
}

March 30, 1995

\begin{abstract}
It is conjectured that transport by parallel mass flow in a braided magnetic field, rather than hyper-resistivity, drives the "dynamo" effect after stochasticity is established. In this paper we do not attempt a rigorous proof of this conjecture, which requires showing that braiding introduces correlations analogous to those giving rise to the neoclassical bootstrap current. We do offer plausible arguments for the conjecture and show that it leads to interesting consequences if true. Namely, magnetic fluctuations would then scale with the magnetic Reynolds number $\mathrm{S}$ like $\widetilde{\mathrm{B}} / \mathrm{B} \sim \mathrm{S}^{-1 / 2}$ and the Rechester-Rosenbluth thermal diffusivity like $\chi_{\mathrm{e}} \propto \mathrm{S}^{-1}$. This scaling would explain the highest temperatures obtained in the CTX spheromak. It also suggests that a "fully-bootstrapped" current drive experiment could be carried out on the DIII-D tokamak.
\end{abstract}

\subsection{Introduction}

An earlier attempt to explain temperatures achieved in the CTX spheromak raised an intriguing question concerning the scaling of magnetic fluctuations, $\widetilde{B}$, with the magnetic Reynolds number, $S .1,2$ On the one hand, evidence from RFP's seems to link transport to tearing modes, ${ }^{3}$ for which Strauss finds $\tilde{B} / B \sim S^{-1 / 3}$ in the non-linear hyper-resistive regime. ${ }^{4}$ On the other hand, there is evidence for $\widetilde{\mathrm{B}} / \mathrm{B}=1.87 \mathrm{~S}^{-1 / 2}$ in $\mathrm{RFP}^{\prime} \mathrm{s}$ and assuming this same value for spheromaks gives a much more reasonable fit to CTX than does the hyper-resistive scaling. 5 
Alfven waves, rather than tearing modes, would give $\tilde{B} / B \simeq S^{-1 / 2}$ as shown in Ref. 1, and tearing modes can cascade to Alfven-like modes of shorter wavelength.6,7 However, the evidence from Ref. 3 for the correlation of transport with tearing modes rather than the cascade causes us to seek a different explanation. Resolving this issue would increase our confidence in predicting spheromak behavior. Moreover, $\mathrm{s}^{-1 / 2}$ scaling is more favorable to achieving ohmic ignition in spheromaks, as discussed in Ref. 2, and it favors more rapid penetration of bootstrap current to the magnetic axis in tokamaks.

\subsection{Parallel Transport}

Perhaps the best theoretical model for transport by low-frequency magnetic turbulence is that of Rechester and Rosenbluth, 8 who find an electron thermal diffusivity:

$$
\chi_{\mathrm{e}}=\mathrm{v}_{\mathrm{e}} \mathrm{L}_{\mathrm{c}} \frac{\tilde{\mathrm{B}}^{2}}{\mathrm{~B}^{2}}
$$

where $v_{e}$ is the electron thermal speed, $L_{c}$ is a correlation length, and $\tilde{B}$ and $B$ are the magnetic fluctuation and average field, respectively. The fitting to CTX discussed above consists of inserting Eq. (1) into the heat balance,

$$
-\nabla \cdot n \chi_{e} \nabla T=\eta j^{2}
$$

with the result

$$
\beta \frac{\tilde{B}^{2}}{B^{2}} \cong \frac{v_{A}}{v_{e}} \frac{R}{L_{c}} S^{-1}
$$


Here $\beta$ is the usual pressure parameter, $v_{A}$ is the Alfven speed, and $R$ is the flux conserver radius. Since $L_{c}$ is unknown, Eq. (3) is used to calculate $L_{c} / R$ from experimental parameters, taking either $\widetilde{B} / B=S^{-1 / 3}$ (the hyper-resistive estimate) or $\widetilde{B} / B=S^{-1 / 2}$. By far the more reasonable value of $L_{C}$ is obtained for $\widetilde{\mathrm{B}} / \mathrm{B}=\mathrm{S}^{-1 / 2}$, which fits two available cases $(\mathrm{R}=.6 \mathrm{~m}, \mathrm{~T}=100 \mathrm{eV}$ and $\mathrm{R}=.3 \mathrm{~m}, \mathrm{~T}=$ $400 \mathrm{eV}$ ) with a single value $L_{c} / R=0.3 .^{5}$ (Both of these cases are collisionless, with $S=10^{5}$ to $10^{6}$, in which case we expect $L_{C} / R \simeq \pi,^{8}$ whereas forcing a fit with $\tilde{B} / B=S^{-1 / 3}$ implies $L_{C} / R \leq .01$.) The actual value used in Ref. 5 is $\tilde{B} / B=1.87$ $\mathrm{S}^{-1 / 2}$ obtained from RFP data. 9

The corresponding particle diffusion coefficient is similar to Eq. (1), with $v_{e}$ replaced by $\mathrm{v}_{\mathrm{i}}$ since electrons cannot leak faster than ions,

$$
D=2 v_{i} L_{c} \frac{\tilde{B}^{2}}{B^{2}}
$$

The factor 2 accounts approximately for the ambipolar linkage of ion and electron fluxes. A particle diffusion rate like Eq. (4) has been observed in RFP's. ${ }^{10}$

\subsection{The Dynamo}

We now turn to the dynamo effect and its relation to magnetic fluctuation levels. One dynamo-type term is $\overrightarrow{\mathrm{u}} \times \overrightarrow{\mathrm{B}}>$ obtained upon averaging ohm's law over fluctuations:

$$
E_{0}+\langle\vec{u} \times \vec{B}>=\eta j
$$

Recall that $\vec{u}$ is the mass velocity, 


$$
\vec{u} \simeq \vec{u}_{i}=n^{-1} \int d \vec{v} f_{i} \vec{v}
$$

The hyper-resistive dynamo is of the order

$$
<u \times B>\simeq<\tilde{u}_{1} \times \tilde{B}_{1}>\cong \frac{\gamma}{k_{\perp}} \frac{\widetilde{B}^{2}}{B}
$$

where $\tilde{u}_{1}$ is the solution of

$$
-\gamma A_{z}+u_{1 x} B_{y o} \cong 0
$$

with $\gamma \tau_{\mathrm{A}} \cong \widetilde{\mathrm{B}} / \mathrm{B}$ and $\tau_{\mathrm{A}} \cong \mathrm{R} / \mathrm{v}_{\mathrm{A}} .1,4$ It is the extra factor of $\tilde{\mathrm{B}}$ through the hyperresistive $\gamma$ that gives $\widetilde{B} / B \sim S^{-1 / 3}$ at saturation. 1,4

Applying this same quasi-linear analysis to mass transport, we obtain

$$
\frac{\partial \rho_{\mathrm{o}}}{\partial \mathrm{t}}+\nabla \cdot<\rho_{1} \tilde{\mathrm{u}}_{1}>=0
$$

where, using the linearized solutions for $\rho_{1}$ and $\tilde{u}_{1}$,

$$
<\rho_{1} \tilde{\mathrm{u}}_{1}>=<\tilde{\mathrm{u}}_{1} \frac{1}{\gamma} \tilde{\mathrm{u}}_{1}>\cdot \nabla \rho_{\mathrm{o}}
$$

or

$$
\mathrm{D}=\left\langle\tilde{\mathrm{u}}_{1} \frac{1}{\gamma} \mathrm{u}_{1}\right\rangle \cong \frac{\gamma}{\mathrm{k}_{\perp}^{2}} \frac{\tilde{\mathrm{B}}^{2}}{\mathrm{~B}^{2}}
$$

Again, near saturation it is the hyper-resistive $\gamma \tau_{\mathrm{A}} \sim \mathrm{S}^{-1 / 3}$ that applies. 
For any interesting value of $v_{i}$, the Rechester-Rosenbluth $D$ in Eq. (4) exceeds the hyper-resistive $D$ in Eq. (11) by a large factor, since $\gamma \sim S^{-1 / 3}$ is very small. Indeed, the Rechester-Rosenbluth $D$ assumes that $\tilde{B}$ is static, as if $\dot{\gamma} \sim 0$. If some kind of Onsager symmetry holds, one suspects that taking the hyper-resistive dynamo given by Eq. (7), but particle transport given by the RechesterRosenbluth rate, Eq. (4), is inconsistent. Particle transport like Eq. (11) implies

$$
u_{x}=-D\left(n-1 \frac{\partial n}{\partial x}\right)
$$

from which

$$
u_{x} B_{y o}=-D\left(n-1 \frac{\partial n}{\partial x}\right) B_{y o} \cong \alpha \frac{2 v_{i} L_{C}}{a} \frac{\tilde{B}^{2}}{B^{2}} B_{y o} .
$$

where $\alpha$ is an undetermined parameter. This expression is much larger than the hyper-resistive dynamo in Eq. (2), again because $\gamma \sim S^{-1 / 3}$ is very small. The saturation fluctuation level is also different (actually smaller), given by

$$
\mathrm{u}_{\mathrm{x}} \mathrm{B}_{\mathrm{yo}}=\eta \mathrm{j}_{\mathrm{z}}
$$

or, using Eq. (13),

$$
\overline{\mathrm{B}}=\left(\frac{\mathrm{v}_{\mathrm{A}}}{2 v_{\mathrm{i}}} \frac{\mathrm{a}}{\mathrm{L}_{\mathrm{c}}}\right)^{1 / 2} \mathrm{~S}^{-1 / 2} \cong \beta_{\mathrm{i}}{ }^{-1 / 4} \mathrm{~S}^{-1 / 2}
$$

This estimate applies to the typical circumstance in which ohmic current drive, or helicity injection, or ohmic decay, continuously drives the system away from the stable Taylor state over a large portion of the radial current profile. Since 
typically $\beta_{\mathrm{i}} \sim .05-.1, \beta_{\mathrm{i}}{ }^{1 / 4}$ is order unity and Eq. (15) predicts $\tilde{B} / \mathrm{B} \sim \mathrm{S}^{-1 / 2}$, consistent with the RFP data of Ref. 9 and with our fitting of CTX discussed above.

Note that Eq. (3) implies a relatively fixed value of $\beta$ that is smaller than unity, consistent with CTX and RFP results in the (collisionless) high temperature regime to which Eq. (3) applies. Equation (3) is an ohmic heating limit on $\beta$, not a pressure-driven instability limit. That the heating limit on $\beta$ is less than unity arises from the fact that $\chi_{\mathrm{e}}$ in the heat transport equation is much larger (by a factor $v_{e} / v_{i}$ ) than $D$ appearing in the current transport, Eq. (13). For theories in which $D$ (mass) $=\chi_{e}$, Eq. (3) would allow $\beta \simeq 1$ in RFP and spheromak, thereby always driving the system to pressure-driven instability, contrary to experience. The hyper-resistive $\chi=\mathrm{D}$ is such a theory.

The kinetic "dynamo" discussed above is better thought of as a fluctuationdriven bootstrap current. For the neoclassical case, Eq. (14). follows from symmetry in the $z$ (toroidal) direction (whereas the even larger poloidal $u_{x} B_{0 z}$ term is balanced by poloidal pressure variations appearing in the generalized ohm's law). ${ }^{11}$ If symmetry also eliminates toroidal variations in pressure and electrostatic potential in the braided field, Eq. (14) would again apply with $u_{x}$ given by Rechester-Rosenbluth transport.

Unlike the corresponding neo-classical bootstrap term, Eq. (13) is not proportional to $\eta$ but rather it contains a variable factor $v_{i} \widetilde{B}^{2}$ that can grow to large values. Thus our fluctuation-driven bootstrap current can be large even if beta-poloidal is small, as it is in spheromaks. On the other hand, being proportional to the poloidal field that vanishes at the magnetic axis, our bootstrap current, like the neo-classical bootstrap, must be supplemented, but 
only very near the axis since the fluctuation bootstrap can be abnormally large off-axis.

Fortunately, Rechester-Rosenbluth transport produces yet another kinetic dynamo effect which, though weaker than the bootstrap, does persist to the axis. This is the viscous current diffusion proposed by Jacobson and Moses. 6 Including both effects yields a toroidal (z-direction) ohm's law with the bootstrap term $(\propto D)$ :

$$
E_{0 z}-D\left(n^{-1} \frac{\partial n}{\partial x}\right) B_{y o}-\frac{4 \pi}{\omega_{p e}^{2}} \frac{\partial}{\partial x} \chi_{e} \frac{\partial j_{z}}{\partial x}-\eta j_{z}=0
$$

This expression would be correct for a neo-classical D. 13

\subsection{Application to Tokamaks}

Boozer has proposed that the dynamo mechanism be employed to transport neoclassical bootstrap current to the magnetic axis in order to achieve a "fullybootstrapped" tokamak. ${ }^{13,14}$ A critical issue in order to test this concept is the time scale for current propagation compared to the duration of experiments.

To carry out a fully-bootstrapped experiment on DIII-D requires current propagation acros's a dimension $\mathrm{a}=0.7 \mathrm{~m}$ in a second or less. Roughly the transport time is, for our kinetic "bootstrap",

$$
\mathrm{t} \sim \frac{\mathrm{a} 2}{4 \mathrm{D}}=\frac{\mathrm{a} 2}{8 \mathrm{v}_{\mathrm{i}} \mathrm{L}_{\mathrm{c}}}\left(\beta_{\mathrm{i}}^{1 / 2} \mathrm{~S}\right)
$$

where we have used Eqs. (4), (13) and (15). For DIII-D we find, crudely, $t \sim 1 \mathrm{sec}$ (for $\beta_{\mathrm{i}}=.01, \mathrm{~S}=10^{8}, \mathrm{v}_{\mathrm{i}}=10^{6} \mathrm{~ms}^{-1}, \mathrm{~L}_{\mathrm{c}}=\mathrm{a}$ ). This would indicate a marginal 
capability to do a fully-bootstrapped experiment in DIII-D. A measurable fraction of bootstrap current has been produced in DII-D in earlier experiments. A more accurate estimate requires joining the Jacobson-Moses transport to the bootstrap near the magnetic axis, as discussed in Sec. 3, Eq. (16).

Incidentally, applied to tokamaks, Eqs. (3) and (15) would imply a heating limit on the poloidal $\beta \ll 1.1,2$ That $\beta_{\text {pol }} \simeq 1$ is actually achieved in ohmically-heated tokamaks gives evidence for good magnetic surfaces and no dynamo effect. To invoke a dynamo effect in tokamak, Boozer calls upon the hollow current profile that would arise in the absence of external current drive in order to excite tearing modes that give rise to the dynamo.

\subsection{Bootstrap Formalism}

The above discussion is only suggestive and requires proof to justify the existence of the Rechester-Rosenbluth bootstrap term.

The formally correct procedure is the Generalized Balescue-Lenard (gBL) theory, ${ }^{15}$ which extends Kaufman's quasi-linear theory for transport in the space of adiabatically conserved action variables $\vec{J}=\left(J_{1}, J_{2}, J_{3}\right) .16$ The gBL theory adds the drag term to give the appropriate Fokker-Planck equation including collisions and fluctuations simultaneously,

$$
\frac{\partial f_{0}}{\partial t}=\frac{\partial}{\partial J} \cdot\left(D \cdot \frac{\partial f_{0}}{\partial J}-\vec{F} f_{0}\right)
$$

The theory automatically exhibits Onsager symmetry. $15,16,17$ 
With simplifying assumptions, Mynick and Duvall have successfully used the gBL theory to derive neoclassical and Rechester-Rosenbluth transport from the same formalism. 17

The Mynick-Duvall results for magnetic turbulence transport do include terms that appear to be analogous to those that generate the neo-classical bootstrap current. ${ }^{18}$ Even so, since their results depend on yet another conjecture (a "pseudo-thermal" spectrum), one would desire corroboration by identifying in braided fields the microscopic physical processes that generate the current. According to Bernstein and Molvig, 18 the neo-classical bootstrap current arises from the fact that, when passing particles moving in opposite directions along the field are scattered toward the trapped state, they move oppositely in radius. Thus, given a density gradient, a current can be created when an excess of $(-)$ directed particles on an inner flux surface and fewer $(+)$ directed particles on an outer surface scatter toward the same flux surface lying between the two original locations of the particles. The magnetic fluctuation-generated bootstrap current postulated in this paper would require that analogous correlations of scattering events be created by magnetic braiding. One such mechanism is discussed in Appendix A.

We note that, unlike the Jacobson-Moses current viscosity term, the RechesterRosenbluth bootstrap term may not conserve helicity. While Bhattacharjee and Hameiri have shown that, within reduced MHD theory, the time-averaged $<\mathrm{V} \times \mathrm{B}\rangle$ term does conserve helicity, ${ }^{19}$ and Boozer has shown that a helicityconserving transport term should have divergence form (like the current viscosity term), 20 the Rechester-Rosenbluth bootstrap based on particle collisional correlations in a static braided magnetic field may not be included in these theories. ${ }^{21}$ The new effect, analogous to the neoclassical theory mentioned 
above and missing from the MHD theory, is a pressure tensor force $\mathrm{F}_{\mathrm{y}}$ appearing in the $y$ (poloidal) ohm's law equation:

$$
-\frac{\partial A_{y}}{\partial t}+F_{y}-u_{x} B_{z}=\eta j
$$

With signs chosen to make $K>0$, the helicity $K$ is then driven by

$$
\frac{d K}{d t}=\int 2 F_{y} B_{y}-\int 2 \eta \vec{j} \cdot \vec{B}
$$

Finally, unlike the neo-classical bootstrap driven by the ever-present thermal fluctuations, the Rechester-Rosenbluth bootstrap would cease to exist in a stable Taylor state decaying classically and therefore by itself it cannot be depended on to drive a steady state, some portion of which would invariably attain a stable profile.

\section{Acknowledgements}

I would like to thank Don Pearlstein and Jim Hammer for clarifying discussions. 


\section{References}

1. T. K. Fowler, "Heat Loss by Helicity Injection in Spheromaks," UCRL-ID116975, March 17, 1994.

2. T. K. Fowler, "Theoretical Aspects of Energy Confinement in Spheromaks," to appear in Fus. Tech.

3. S. Prager, private communication. See also G. Fiksel, et al., Phys. Rev. Ltr 72, 1028 (1994).

4. H. R. Strauss, Phy. Fluids 29,3668 (1986).

5. E. B. Hooper, J. H. Hammer, C. W. Barnes and J. C. Fernandez, "A Reexaminatioin of Spheromak Experiments and Opportunities," to appear in Fus. Tech.

6. N. Mattor, P. W. Terry and S. C. Prager, Comments on Plasma Physics and Controlled Fusion, 15, 65 (1992).

7. D. Biskamp, Plasma Phys. and Controlled Fusion, 26, 311 (1984).

8. A. B. Rechester and M. N. Rosenbluth, Phys. Rev. Letters 40,38 (1978).

9. R. L. Lahaye, T. N. Carlstrom, R. R. Goforth, G. L. Jackson, M. J. Shaffer, T. Tamano, and P. L. Taylor, Phys. Rev. Letters 27,2576 (1984).

10. M. R. Stoneking, S. A. Hokin, S. C. Prager, G. Fiksel, H. Ji and D. J. Den

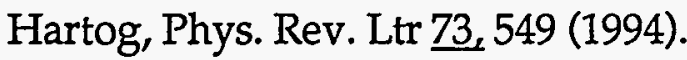

11. F. L. Hinton and R. D. Hazeltine, Rev. Mod. Phys. 48,239 (1976).

12. Abram R. Jacobson and Ronald W. Moses, Physical Review A, 29, 3335 (1984).

13. R. H. Weening and A. H. Boozer, Phys. Fluids B $\underline{4}, 159$ (1992).

14. A. H. Boozer, Phys. Fluids 29,4132 (1986).

15. H. E. Mynick, J. Plasma Phys. 39 , 303 (1988).

16. A. N. Kaufman, Phys. Fluids 15,1063 (1972). See also I. B. Bernstein and K. Molvig, Phys. Fluids 26, 1488 (1983), and R. H. Cohen, K. Hizardis, K. Molvig and I. B. Bernstein, Phys. Fluids 27 377 (1984). 
17. H. E. Mynick and R. E. Duvall, Phys. Fluids B 1,750 (1988). See also, H. E. Mynick, Nuclear Fusion 30, 357 (1990).

18. I. B. Bernstein and K. Molvig, Phys. Fluids 26 , 1488 (1983).

19. A. Bhattacharjee and E. Hameiri, Phys. Rev. Letters 57, 206 (1986).

20. A. H. Boozer, J. Plasma Phys. $\underline{35} 133$ (1986).

21. J. A. Krommes, C. Oberman and R. G. Kleva, J. Plasma Phys. 30 , 11 (1983).

22. W. J. Nunan and J. M. Dawson, Phys. Rev. Ltrs, 73, 1628 (1994); S. Ma and J. M. Dawson, Phys. Plasmas, 1, 12511251 (1994).

23. K. C. Shiang, Phys. Fluids B 2, 764 (1990) and references therein. 


\section{Appendix A}

Further light can be shed on the origin of bootstrap current by again considering the ohm's law for average field quantities:

$$
-\frac{\partial \vec{A}}{\partial t}=\eta \vec{j}-\overrightarrow{F_{e}}-\overrightarrow{u_{e}} \times \vec{B}
$$

where

$$
\begin{aligned}
& \overrightarrow{F_{e}}=(n e)^{-1}\left(\nabla \cdot P_{e}-\text { ne } \nabla \phi\right)+\left\langle u_{1} \times B_{1}\right\rangle \\
& \vec{u}_{e} \cong B^{-2}\left(-\frac{\partial \vec{A}}{\partial t} \times \vec{B}\right)+\left(-p^{-1} D \nabla p\right)+\vec{u}_{D} \\
& D=D_{C}+D_{N C} .
\end{aligned}
$$

Here $P_{e}$ is the electron stress tensor, $p$ is the scalar pressure, $D_{c}=v_{e i} r_{L e}{ }^{2}$ is the classical diffusion coefficient, $D_{N C}$ is any non-classical part, and $\vec{u}_{D}$ is the diamagnetic term that balances the radial component of $\vec{F}_{e}$, of no further interest.

For $\mathrm{D}_{\mathrm{NC}}$, we consider only neo-classical transport, 11 or Rechester-Rosenbluth transport in a static braided field, 8 in which case we may take $\eta$ to be classical (or neo-classical). We have, however, retained a possible Rechester-Rosenbluth contribution to $\left\langle u_{1} \times B_{1}\right\rangle$ averaged over microscopic motion of electrons in the braided field.

For a classical $\eta$, the $j_{\perp}$ and $D_{c}$ terms cancel in Eq. (A1), leaving 


$$
\begin{aligned}
& -\frac{\partial A \|}{\partial t}=\eta j \|-F_{e} \\
& F_{e \perp}=D_{N C} p^{-1}(\nabla p \times \vec{B})_{\perp}
\end{aligned}
$$

where $\perp$ means perpendicular to $\vec{B}$ but lying in the flux surface. Thus the existence of $D_{N C}$ demands the existence of a force $F_{e \perp}$, but the bootstrap follows only if this force contributes a parallel component in Eq. (A5). For neo-classical transport, caused by toroidal bending of the plasma column, $\overrightarrow{\mathrm{F}_{\mathrm{e}}}$ is purely poloidal by symmetry, from which we find

$$
F_{e \|}=\frac{B_{\theta}}{B_{Z}} F_{e \perp} \cong D_{N C} \frac{B_{\theta}}{p} \frac{d p}{d r}
$$

(using $B_{\theta} \ll B_{Z}$ as in tokamaks). Substituting banana diffusion,

$$
D_{\mathrm{NC}}=\sqrt{\varepsilon} \rho_{\theta \mathrm{e}^{2}} v_{\mathrm{ei}}
$$

gives $F_{e \| l}=\eta$ jв where $j_{B}$ is the familiar tokamak bootstrap current.11 This approximate result follows solely from force balance, symmetry and the heuristic .. estimate of $D_{N C}$ for neo-classical transport, without detailed knowledge of $\vec{F}_{\mathrm{e}}$.

From this example we see that the conjecture in this paper requires that $\vec{F}_{e}$ again be purely poloidal for transport in a braided field. The neo-classical argument based on toroidal symmetry no longer applies, since magnetic braiding (due to tearing, etc.) could occur even in a straight cylinder. However, on closer inspection we see that braiding also implies poloidal distortions since braiding can be regarded as a superposition of tiny discharge filaments, each of which is twisted poloidally in the same sense in its attempt to follow the helical lines of the average field. Then we can again estimate the bootstrap current from Eq. (A7) 
with $D_{\mathrm{NC}}$ given by Rechester-Rosenbluth mass transport, the result being equivalent to Eq. (14) of the main text.

Using Mynick's theory ${ }^{15}$ (our Eq. (17)), we find that, for Rechester-Rosenbluth transport, his $\mathrm{D}$ term produces the $\overrightarrow{u_{N}} \mathrm{C} \times \overrightarrow{\mathrm{B}}$ term of the ohm's law. This implies that his friction term $\vec{F}$ corresponds to $\vec{F}_{\mathrm{e}}$ in the ohm's law, $\overrightarrow{\mathrm{F}_{\mathrm{e}}}$ being required to cancel $\overrightarrow{\mathrm{u}}_{\mathrm{NC}} \times \overrightarrow{\mathrm{B}}$. Whether or not there is a residual $\overrightarrow{\mathrm{Fe}}_{\|}$to drive the bootstrap in Mynick's theory is a more subtle question not resolved at this writing. However, we note that Mynick has shown that his $\vec{F}$ term does drive an inward particle pinch, 22 which Bernstein and Molvig found to be the process that is Onsager-symmetric to the boostrap current in the neoclassical case. ${ }^{18}$ From this we tentatively infer that it is Mynick's friction - actually nonlocal in the sense that one must average over fluctuation radial wavelengths ${ }^{17}$ that provides the necessary correlations to produce a current.

Dawson $^{22}$ and Shiang 23 have found similar bootstrap currents driven by electric field turbulence. $\mathrm{Ma}$ and Dawson 22 conclude that the effect is inceed due to symmetry based on general considerations which, if correct, would apply also to the magnetic turbul'ence bootstrap postulated here. 